\title{
Bounds of Proof Complexities in Some Systems for Many- Valued Logics
}

\author{
Arman Tshitoyan \\ PhD Student, Department of Informatics and Applied Mathematics, Yerevan State University, Yerevan, \\ Armenia \\ Email: tsh_arman@yahoo.com
}

\begin{abstract}
In this paper the main proof complexity characteristics in two types of proof systems for a version of many valued propositional logic are investigated for some class of $k$-valued $(k \geq 3)$ tautologies. We consider a Hilbert style cut free system and a system, which is dual to resolution system, for many valued logic with implication, defined by Gödel, and negation, defined by permuting the truth values cyclically. For considered class of tautologies we obtain simultaneously optimal bounds for different proof complexity measures (asymptotically the same upper and lower bounds for each measures).
\end{abstract}

Keywords: Many-valued logics, Hilbert style proof systems, sequent proof systems, elimination systems, proof complexity.

\section{Introduction}

Many-valued logic (MVL) as a separate subject was created and developed first by Łukasiewicz [1]. Later on many others continued investigation in this area. In the earlier years of development, this caused some doubts about the usefulness of MVL. In the meantime, however, many interesting applications were found in such fields as logic, mathematics, hardware design, artificial intelligence and some other area soft information technologies, therefore the investigations in area of proof complexity for different systems of MVL are very important.

In this paper the main proof complexity characteristics in two types of proof systems for a version of many valued propositional logic are investigated for some class of $k$-valued $(k \geq 3)$ tautologies. For many valued logic with implication, defined by Gödel, and negation, defined by permuting the truth values cyclically, we consider the "elimination" system, which is based on the determinative disjunctive normal form, introduced in [2,3], and a Hilbert style cut-free system, defined in [4]. We suggest some generalization for a family of 2-valued tautologies, which are known as "hard" for some proof systems of classical propositional logic tautologies. For introduced class of tautologies we obtain simultaneously optimal bounds for different proof complexity measures (asymptotically the same upper and lower bounds for each measures).

Short presentation of main results only for one from mentioned systems was in [3].

\section{$2 \quad$ Preliminaries}

Here we give some of well-known notions and notations in area of MVL.

\section{$2.1 \quad$ k-valued Logic}

Let $E_{k}$ be the set $\left\{0, \frac{1}{\mathrm{k}-1}, \ldots, \frac{\mathrm{k}-2}{\mathrm{k}-1}, 1\right\}$. We use the well-known notions of propositional formula, which, defined as usual from k-valued propositional variables $p, q, p_{i}(i \geq 1)$ with values from $E_{k}$, may be also propositional constants, parentheses, and logical connectives $\wedge, \vee, \supset, \neg$ defined as follows:

$$
p \vee q=\max (p, q),
$$




$$
\begin{aligned}
& p \wedge q=\min (p, q), \\
& p \supset q= \begin{cases}1, \text { for } p \leq q \\
q, \text { for } p>q\end{cases}
\end{aligned}
$$

and cyclically permuting negation, defined as follow:

$$
\neg p=(((k-1) p+1) \bmod k) /(k-1) .
$$

For negation we use also denotation $\bar{p}$.

For propositional variable $p$ and $\delta=\frac{i}{\mathrm{k}-1}(0 \leq i \leq k-1)$ we define additionally exponent-function $p^{\delta}$ as $p$ with $(k-1)-i$ negations.

In considered logic we fix 1 as designated value, so a formula $\varphi$ with variables $p_{1}, p_{2}, \ldots, p_{n}$ is called $k$-tautology if for every $\tilde{\delta}=\left(\delta_{1}, \delta_{2}, \ldots, \delta_{n}\right) \in E_{k}^{n}$, assigning $\delta_{j}(1 \leq j \leq n)$ to each $p_{j}$ gives the value 1 for $\varphi$.

Our investigations will be focused on $\mathrm{k}$-valued $(k \geq 3)$ logics, but sometimes for simplification of consideration we demonstrate the main results only for the 3 -valued logics.

\subsection{Determinative Disjunctive Normal Form}

Here we recall the notions of determinative conjunct and determinative disjunctive normal form, introduced by A. Chubaryan for 2-valued Boolean functions in [2] and generalized in [3] for 3-valued logic.

Here we will use the current concepts of the unit Boolean cube $\left(E^{n}\right)$ for $E=\{0,1\}$, a propositional formula and a classical tautology. The particular choice of a language for presented propositional formulas is immaterial in this consideration. However, because of some technical reasons we assume that the language contains the propositional 2 -valued variables $p_{i}(i \geq 1)$ and(or) $p_{i_{j}}(i \geq 1 ; j \geq 1)$, logical connectives $\wedge, \vee, \supset, \neg$ and parentheses. Following the usual terminology we call the variables and negated variables literals for 2-valued logic. The conjunct K (term) can be represented simply as a set of literals (no conjunct contains a variable and its negation simultaneously), and disjunctive normal form $(D N F)$ can be represented as a set of conjuncts.

In $[2]$ the following notions were introduced.

We call a replacement-rule each of the following trivial identities for a propositional formula $\psi$ :

$$
\begin{array}{lccc}
0 \wedge \psi=0, & \psi \wedge 0=0, & 1 \wedge \psi=\psi, & \psi \wedge 1=\psi, \\
0 \vee \psi=\psi, & \psi \vee 0=\psi, & 1 \vee \psi=1, & \psi \vee 1=1, \\
0 \supset \psi=1, & \psi \supset 0=\bar{\psi}, & 1 \supset \psi=\psi, & \psi \supset 1=1, \\
\overline{0}=1, & \overline{1}=0, & \psi=\psi, & \\
0 \equiv \psi=\bar{\psi}, & \psi \equiv 0=\bar{\psi}, & 1 \equiv \psi=\psi, & \psi \equiv 1=\psi .
\end{array}
$$

Application of a replacement-rule to some word consists in replacing of its subwords, having the form of the left-hand side of one of the above identities, by the corresponding right-hand side.

Let $\varphi$ be a propositional formula, $P=\left\{p_{1}, p_{2}, \ldots, p_{n}\right\}$ be the set of all variables of $\varphi$, and $P^{\prime}=\left\{p_{i_{1}}, p_{i_{2}}, \ldots, p_{i_{m}}\right\}(1 \leq m \leq n)$ be some subset of $P$.

Definition 1: Given $\sigma=\left\{\sigma_{1}, \sigma_{2}, \ldots, \sigma_{m}\right\} \subset E^{m}$, the conjunct $K^{\sigma}=\left\{p_{i_{1}}{ }^{\sigma_{1}}, p_{i_{2}}{ }^{\sigma_{2}}, \ldots, p_{i_{m}}{ }^{\sigma_{m}}\right\}{ }^{1}$ is called $\varphi-1$-determinative $\left(\varphi-0\right.$-determinative) if assigning $\sigma_{j}(1 \leq j \leq m)$ to each $p_{i j}$ and successively

${ }^{1}$ As usual, given a propositional variable $p$ and $\sigma \in E^{1}$, by $p^{\sigma}$ we denote the function $p^{\sigma}=\left\{\begin{array}{l}p, \text { if } \sigma=1 \\ \bar{p}, \text { if } \sigma=0\end{array}\right.$ 
using replacement-rules we obtain the value of $\varphi$ (1 or 0) independently of the values of the remaining variables.

$\varphi-1$-determinative and $\varphi-0$-determinative conjuncts are called also $\varphi$-determinative or determinative for $\varphi$.

Definition 2: A DNF $D=\left\{K_{1}, K_{2}, \ldots, K_{j}\right\}$ is called determinative DNF (dDNF) for $\varphi$ if $\varphi=D$ and every conjunct $K_{i}(1 \leq i \leq j)$ is 1-determinative for $\varphi$.

The notion of determinative disjunctive normal form for 3-valued logic is generalized in [3] as follows.

Above mentioned replacement-rules are valid for $\wedge$ and $\vee$, but for $\supset$ are valid only $0 \supset \psi=1,1 \supset \psi=\psi$ and $\psi \supset 1=1$. For negation the replacement rules are $\neg 0=1 / 2, \neg 1 / 2=1$, $\neg 1=0$ and $\neg \neg \neg \psi=\psi$.

For the other cases we have introduced the following auxiliary relations for replacement

$$
\begin{aligned}
& 1 / 2 \wedge \psi=\psi \wedge 1 / 2 \leq 1 / 2,1 / 2 \vee \psi=\psi \vee 1 / 2 \geq 1 / 2, \\
& \psi \supset 0=\neg s g \psi, \quad 1 / 2 \supset \psi=s g \psi, \quad \psi \supset 1 / 2 \geq 1 / 2,
\end{aligned}
$$

where by $\neg s g \psi$ is denoted a function, which is equal to 0 , if value of $\psi$ is more than 0 , and 1 in the opposite case, and by $s g \psi$ is denoted the function, which is equal to 1 , if value of $\psi$ is more than 0 , and 0 , in the opposite case.

For every propositional variable $p$ in 3-valued logic $p, \neg p$ and $\neg \neg p$ are the literals.

Let $\varphi$ be a propositional formula of 3 -valued logic, $P=\left\{p_{1}, p_{2}, \ldots, p_{n}\right\}$ be the set of all variables of $\varphi$ and $P^{\prime}=\left\{p_{i_{1}}, p_{i_{2}}, \ldots, p_{i_{m}}\right\}(1 \leq m \leq n)$ be some subset of $P$.

Definition 3. Given $\tilde{\sigma}=\left(\sigma_{1}, \sigma_{2}, \ldots, \sigma_{m}\right) \in E_{3}^{m}$, the conjunct $K^{\sigma}=\left\{p_{i_{1}}{ }^{\sigma_{1}}, p_{i_{2}}{ }^{\sigma_{2}}, \ldots, p_{i_{m}}{ }^{\sigma_{m}}\right\}$ is called $\varphi-1$ -determinative ( $\varphi-0$-determinative, $\varphi-1 / 2$-determinative), if assigning $\sigma_{j}(1 \leq j \leq m)$ to each $p_{i_{j}}$ and successively using replacement-rules and, if it is necessary, the auxiliary relations for replacement also, we obtain the value of $\varphi(1,0,1 / 2)$ independently of the values of the remaining variables.

For example the conjuncts $p_{1}, \neg p_{1}$ and $\neg \neg p_{1}$, are 1-determinative for the formula $p_{1} \supset\left(p_{2} \supset\left(p_{3} \supset p_{1}\right)\right)$. For the first two we use only replacement-rules, but for the last one we must use the auxiliary relations for replacement also.

Definition of $d D N F$ for 3 -valued logic is given by analogy.

Remark 1. It is easily proved that

1) If for some tautology $\varphi$, the minimal number of literals, containing in $\varphi$-determinative conjunct, is $m$, then $\varphi$-determinative $D N F$ has at least $3^{m}$ conjuncts;

2) If for some tautology $\varphi$ there is such $m$ that every conjunct with $m$ literals is $\varphi$-determinative, then there is $\varphi$-determinative $D N F$ with no more than $3^{m}$ conjuncts.

By analogy we can define the determinative conjuncts and $d D N F$ for $\mathrm{k}$-valued logic with the mentioned properties. For k-valued logic we must introduce the corresponding replacement-rules and auxiliary relations for replacement.

\section{Definitions of Main Systems}

Here we recall the main proof systems following [3, 4]. 


\subsection{Elimination Systems ECN $_{k}$}

The axioms of $E C N_{k}$ aren't fixed, but for every formula $\varphi$ each conjunct from some dDNF of $\varphi$ can be considered as an axiom.

For 3-valued logic the elimination rule ( $\varepsilon$-rule) infers conjunct $K^{\prime} \cup K^{\prime \prime} \cup K^{\prime \prime \prime}$ from conjuncts $K^{\prime} \cup\{p\}, K^{\prime \prime} \cup\{\neg p\}$ and $K^{\prime \prime \prime} \cup\{\neg \neg p\}$, where $K^{\prime}, K^{\prime \prime}$ and $K^{\prime \prime \prime}$ are conjuncts and $p$ is a variable. It is obvious, that this rule can be easily generalized for k-valued logic with cyclical negation.

The proof in $E C N_{k}$ is a finite sequence of conjuncts such that every conjunct in the sequence is one of the axioms of $E C N_{k}$ or is inferred from earlier conjuncts in the sequence by $\varepsilon$-rule.

A DNF $D=\left\{K_{1}, K_{2}, \ldots, K_{l}\right\}$ is tautological if by using $\varepsilon$-rule can be proven the empty conjunct $(\varnothing)$ from the axioms $\left\{K_{1}, K_{2}, \ldots, K_{l}\right\}$.

\subsection{The System $\mathrm{CN}_{3}$-Cut-Free}

The schematic axioms of the system $\mathrm{CN}_{3}$-cut-free are the following

1. $\quad \alpha_{1} \wedge\left(\alpha_{1} \wedge \ldots \wedge\left(\alpha_{m-1} \wedge \alpha_{m}\right) \ldots\right) \supset \alpha_{i}, m \geq 1,1 \leq i \leq m$

2. $\quad$ a. $\left(K \supset \alpha^{\sigma_{1}}\right) \supset\left(\left(K \supset \beta^{\sigma_{2}}\right) \supset\left(K \supset(\alpha \supset \beta)^{\varphi_{\supset}\left(\alpha, \beta, \sigma_{1}, \sigma_{2}\right)}\right)\right)$

b. $\left(K \supset \alpha^{\sigma_{1}}\right) \supset\left(\left(K \supset \beta^{\sigma_{2}}\right) \supset\left(K \supset(\alpha \vee \beta)^{\varphi_{\vee}\left(\alpha, \beta, \sigma_{1}, \sigma_{2}\right)}\right)\right)$

c. $\left(K \supset \alpha^{\sigma_{1}}\right) \supset\left(\left(K \supset \beta^{\sigma_{2}}\right) \supset\left(K \supset(\alpha \wedge \beta)^{\varphi_{\wedge}\left(\alpha, \beta, \sigma_{1}, \sigma_{2}\right)}\right)\right)$

d. $\left(K \supset \alpha^{\sigma_{1}}\right) \supset\left(\left(K \supset \beta^{\sigma_{2}}\right) \supset\left(K \supset\left(\alpha^{\beta}\right)^{\varphi_{\text {exp }}\left(\alpha, \beta, \sigma_{1}, \sigma_{2}\right)}\right)\right.$

e. $\left.\left(K \supset \alpha^{\sigma}\right) \supset(K \supset \neg \alpha)^{\bar{\sigma}}\right)$

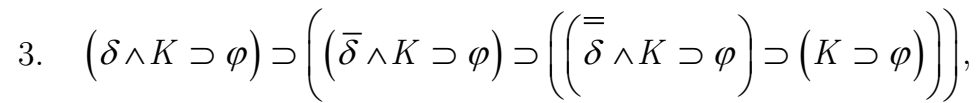

$(\gamma \supset \varphi) \supset((\bar{\gamma} \supset \varphi) \supset(\bar{\gamma} \supset \varphi) \supset \varphi))$

where

1. $\varphi$ is provable formula,

2. $\alpha_{i}(1 \leq i \leq m)$ and $\gamma$ are literals, $\alpha, \beta, \delta$ are arbitrary formulas,

3. $K=\beta_{1} \wedge\left(\beta_{2} \wedge \ldots \wedge\left(\beta_{l-1} \wedge \beta_{l}\right) \ldots\right),(l \geq 1)$ for arbitrary literals $\beta_{i}(1 \leq i \leq l)$,

4. for every $\beta_{1} \wedge\left(\beta_{2} \wedge \ldots \wedge\left(\beta_{l-1} \wedge \beta_{l}\right) \ldots\right) \supset \psi$ style subformula from some aciom of second group conjuct $\left\{\beta_{1}, \ldots, \beta_{l}\right\}$ is $\psi$-determinable,

5. if $K^{\text {set }}=\left\{\beta_{1}, \ldots, \beta_{n}\right\}$ for some subformula $K=\beta_{1} \wedge \beta_{2} \wedge \ldots \wedge \beta_{k}$ from first axiom of third group, then $\delta \notin K^{\text {set }}$ and $\delta \cup K^{\text {set }}$ is subset of some $\varphi$-determinative conjunct, but $K^{\text {set }}$ is not $\varphi$-determinative.

6. $\varphi_{\supset}\left(A, B, \sigma_{1}, \sigma_{2}\right)=\left(\sigma_{1} \supset \sigma_{2}\right) \wedge(\neg(A \vee \bar{A}) \vee(\overline{\bar{B}} \supset B)) \vee(\neg(A \vee \overline{\bar{A}}) \wedge \neg(B \vee \overline{\bar{B}}))$,

7. $\varphi_{\vee}\left(A, B, \sigma_{1}, \sigma_{2}\right)=\left(\sigma_{1} \vee \sigma_{2}\right) \vee((A \supset \bar{A}) \wedge \neg(\bar{B} \vee \bar{B})) \vee(\neg(\bar{A} \vee \overline{\bar{A}}) \wedge(B \supset \bar{B}))$, 


$$
\begin{aligned}
& \text { 8. } \varphi_{\wedge}\left(A, B, \sigma_{1}, \sigma_{2}\right)=\left(\begin{array}{ll}
\sigma_{1} ? \wedge & \sigma_{2}
\end{array}\right) \vee((A \wedge \bar{A}) \vee(B \wedge \bar{B})) \vee((A \wedge \bar{A}) \vee(B \wedge \bar{B}) \text {, } \\
& \text { 9. } \varphi_{\text {exp }}\left(A, B, \sigma_{1}, \sigma_{2}\right)=\sigma_{1}^{\sigma_{2}} \vee\left(\neg\left(\sigma_{1}^{\sigma_{2}}\right) \wedge \neg\left(\neg\left(A^{\sigma_{1}} \wedge \bar{B}^{\sigma_{2}}\right) \vee \neg \neg\left(A^{\sigma_{1}} \wedge \bar{B}^{\sigma_{2}}\right)\right)\right)
\end{aligned}
$$

Rule of inference is modus ponens

$$
\frac{A \quad A \supset B}{B}
$$

Note that, in spite of rule modus ponens, the restrictions 1.-5. "insist on construction" of derived formula $\varphi$ step by step on the basis of determinative conjuncts.

Note also that the corresponding systems $\mathrm{CN}_{\mathrm{k}}$-cut-free for $k \geq 4$ can be constructed by analogy. Some difficultness for generalization of above systems is the definition of the functions $\varphi_{*}\left(A, B, \sigma_{1}, \sigma_{2}\right)$ for $* \in\{\exp , \wedge, \vee, \supset\}$, as described in the corresponding axioms schemas 2.a) - 2.d) of the system $\mathrm{CN}_{3}$ cut-free.

\section{Proof Complexity Measures}

In the theory of proof complexity two main characteristics of the proof are: $t$-complexity, defined as the number of proof steps (length) and $l$-complexity, defined as total number of proof symbols (size). We consider two measures (space and width) also: $s$-complexity (space), informal defined as maximum of minimal number of symbols on blackboard, needed to verify all steps in the proof and $w$ - complexity (width), defined as the maximum of widths of proof formulas.

Following [5] we give the formal definitions of mentioned proof complexity measures.

If a proof in the system $\Phi$ is a sequence of lines, where each line is an axiom, or is derived from previous lines by one of a finite set of allowed inference rules, then a $\Phi$-configuration is a set of such lines. A sequence of $\Phi$-configurations $\left\{D_{0}, D_{1}, \ldots, D_{r}\right\}$ is said to be $\Phi$-derivation if $D_{0}$ is an empty set and for all $t(1 \leq t \leq r)$ the set $D_{t}$ is obtained from $D_{t-1}$ by one of the following derivation steps:

Axiom Download: $D_{t}=D_{t-1} \cup\left\{L_{A}\right\}$, where $L_{A}$ is an axiom of $\Phi$.

Inference: $D_{t}=D_{t-1} \cup\{L\}$, for some $L$ inferred by one of the inference rules for $\Phi$ from a set of assumptions, belonging to $D_{t-1}$.

Erasure: $D_{t} \subset D_{t-1}$.

A $\Phi$-proof of a tautology $\varphi$ is a $\Phi$-derivation $\left\{D_{0}, D_{1}, \ldots, D_{r}\right\}$ such that $\tilde{\varphi} \in D_{r}$, where $\tilde{\varphi}$ is empty conjunct in $E C N_{k}$ and $\tilde{\varphi}$ is $\varphi$ in $\mathrm{CN}_{\mathrm{k}}$-cut-free.

By $|\varphi|$ we denote the size of a formula $\varphi$, defined as the number of all logical signs entries. It is obvious that the full size of a formula, which is understood to be the number of all symbols is bounded by some linear function in $|\varphi|$.

The size $(l)$ of a $\Phi$-derivation is a sum of the sizes of all lines in a derivation, where lines that are derived multiple times are counted with repetitions. The steps $(t)$ of a $\Phi$-derivation is the number of axioms downloads and inference steps in it. The space $(s)$ of a $\Phi$-derivation is the maximal space of a configuration in a derivation, where the space of a configuration is the total number of logical signs in a configuration, counted with repetitions. The width $(w)$ of a $\Phi$-derivation is the size of the widest line in a derivation.

Let $\Phi$ be a proof system and $\varphi$ be a tautology. We denote by $t_{\varphi}^{\Phi}\left(l_{\varphi}^{\Phi}, s_{\varphi}^{\Phi}, w_{\varphi}^{\Phi}\right)$ the minimal possible value of $t$-complexity $(l$-complexity,, 3 -complexity, $w$-complexity) for all proofs of tautology $\varphi$ in $\Phi$. 


\section{Main Results}

Before we'll prove the main theorem, we must give some auxiliary results.

In some papers in the area of propositional proof complexity for 2-valued classical logic the following tautologies (Topsy-Turvy Matrix) play a key role

$$
T T M_{n, m}=\underset{\left(\sigma_{1}, \sigma_{2}, \ldots, \sigma_{n}\right) \in E^{n}}{\vee} \underset{j=1}{\wedge} \vee_{i=1}^{n} p_{i_{j}}^{\sigma_{j}} \quad\left(n \geq 1,1 \leq m \leq 2^{n}-1\right) .
$$

For all fixed $n \geq 1$ and $m$ in above indicated intervals every formula of this kind expresses the following true statement: given a 0,1-matrix of order $n \times m$ we can "topsy-turvy" some strings (writing 0 instead of 1 and 1 instead of 0 ) so that each column will contain at least one 1.

We generalize this family of 2 -tautologies for $k$-valued $(k \geqslant 3)$ logic.

For given $\tilde{\sigma}=\left(\sigma_{1}, \sigma_{2}, \ldots, \sigma_{m}\right) \in E_{k}^{m}$ and $\delta=\frac{i}{\mathrm{k}-1}(0 \leq i \leq k-1)$ we call $\delta$-topsy-turvy-result the cortege $\widetilde{\sigma \delta}$, which contains every $\sigma_{j}(1 \leq j \leq m)$ with $(k-1)-i$ negations. We say that a cortege is topsy-turvy-result from the other cortege if there is $\delta=\frac{i}{\mathrm{k}-1}$ such, that the first cortege is $\delta$-topsyturvy-result from the second one.

For given $\tilde{\sigma}=\left(\sigma_{1}, \sigma_{2}, \ldots, \sigma_{m}\right) \in E_{k}^{m}$ and $\delta=\frac{i}{\mathrm{k}-1}(0 \leq i \leq k-1)$ we denote by $|\tilde{\sigma}(\delta)|$ the number of $\delta$ occurrence in $\tilde{\sigma}$.

Lemma 1. In given 3-valued $0,1 / 2,1$-matrix of order $n \times m$, we can "topsy-turvy" some strings such, that each column will contain at least one 1 , iff $m$ is no more than $f(n)$, where $f(n)$ is defined as follows:

$$
f(1)=1, f(n+1)=f(n)+[f(n) / 2]+1
$$

Proof is given by induction on number $n$ of matrix strings. For $n=1, m=1$. Suppose that statement is valid for $n$ strings. If the number of strings is $n+1$, we consider the last string. If for some $\delta=\frac{i}{\mathrm{k}-1}(0 \leq i \leq k-1) \quad|\tilde{\sigma}(\delta)| \geq[(\mathrm{m}+2) / 3]$ then after $\delta$-topsy-turvy we obtain in the last string at least $[(\mathrm{m}+2) / 3] 1$, therefore we'll have at least $[(m+2) / 3]$ columns, which contain at least one 1 . For the other columns we consider cortege of $n$-th string and repeat the same action. Let $x$ be the maximum columns, which we can add to matrix by order $(n+1) \times f(n)$, such that we can do "topsy-turvy" on some strings, such that each column of new matrix will contain at least one 1 . For above it is follow, that $f(n+1)=f(n)+x$, where $x=[(f(n+1)=2) / 3]$, therefore we have $x \leq(f(n+1)=2) / 3$, then $f(n+1) \geq 3 x-2$, so $f(n)+x \geq 3 x-2$, from which $f(n) / 2 \geq x-1$ and finally $x=[f(n) / 2]+1$.

Corollary 1. In given $\mathrm{k}$-valued $(\mathrm{k} \geqslant 4)$ matrix of order $n \times m$, we can "topsy-turvy" some strings, such that each column will contain at least one 1 , iff $m$ is no more than $f(n)$, where $f(n)$ is defined as follows:

$$
f(1)=1, f(n+1)=f(n)+[f(n) / k-1]+1
$$

Proof can be given by analogy.

Corollary 2. For every $n \geq 1$ and $m \leq f(n)$ the following formulas are k-tautologies

$$
\left.T T M_{n, m}=\underset{\left(\sigma_{1}, \sigma_{2}, \ldots, \sigma_{n}\right)}{\vee} \underset{j=1}{\stackrel{n}{v_{i=1}^{n}} p_{i_{j}}} \sigma_{j} \text {, (where first disjunctions are for all }\left(\sigma_{1}, \sigma_{2}, \ldots, \sigma_{n}\right) \in E_{k}^{n}\right) \text {, }
$$

Proof is obvious.

Note that $f(n+1)=f(n)+[f(n) / 2]+1 \geq f(n)+f(n) / 2=3 f(n) / 2 \geq \ldots \geq 3^{n} / 2^{n}>3^{[n / 3]}$. 
Corollary 3. For every $n \geq 1$ and $m=3^{[n / 3]}$ the following formulas are 3 -tautologies

$$
\left.T T M_{n, m}=\underset{\left(\sigma_{1}, \sigma_{2}, \ldots, \sigma_{n}\right)}{\vee} \underset{j=1}{\wedge} \underset{i=1}{\vee} p_{i_{j}}^{\sigma_{j}}, \text { (where first disjunctions are for all }\left(\sigma_{1}, \sigma_{2}, \ldots, \sigma_{n}\right) \in E_{3}^{n}\right)
$$

The analogous formulas for $\mathrm{k}$-valued logics are $\mathrm{k}$-tautologies for every $n \geq 1$ and $\mathrm{m}=k^{[n / k]}$.

Lemma 2. The systems $\mathrm{CN}_{3}$ and $\mathrm{CN}_{3}$-cut-free are polynomially equivalent by all proof complexity measures.

Proof. Let us recall that polynomial equivalence means, that transformation of any proof in one system into a proof in the other system can be done with no more than polynomial increase of proof complexity. It is not difficult to see that every proof of empty conjunct from dDNF of any formula $\varphi$ in the system $\mathrm{CN}_{3}$ can be easily transformed into proof of $\varphi$ in the system $\mathrm{CN}_{3}$-cut-free with no more than linear $(*|\varphi|)$ increase of $t, s$ and $w$ complexity and with no more than $\left(*|\varphi|^{2}\right)$ increase of size. Really we can derive formula $\varphi$ from every $\varphi$-determinative conjunct, using the axioms of the first and second groups, and then, using the axioms of the last group, derive $\varphi$. Reverse transformation is simpler. Really we can take as axioms every $\varphi$-determinative conjunct from the first occurrences of axioms 3(a) in proof. It is obvious, that in such reverse transformation we have no increase.

Lemma 3. The bounds of minimal possible value of $s$-complexity for all proofs of 3-tautology $\varphi$ with $n$ variables in $\mathrm{CN}_{3}$ are: $s_{\varphi}=O\left(n^{2}\right)$ and $s_{\varphi}=\Omega(n)$.

Proof. For upper bound we use the perfect DNF D of $\varphi$, which obviously is dDNF.

We consider the following tree like refutation of $\mathrm{D}$ in the system $\mathrm{CN}_{3}$, whereas axioms from the left to the right are the following conjuncts:

$$
p_{1}^{0}, p_{2}^{0}, \ldots, p_{n}^{0} \quad p_{1}^{0}, p_{2}^{0}, \ldots, p_{n}^{1 / 2} \quad p_{1}^{0}, p_{2}^{0}, \ldots, p_{n}^{1} \quad \ldots . . \quad p_{1}^{1}, p_{2}^{1}, \ldots, p_{n}^{1}
$$

Number of conjuncts used as axioms will be $3^{k}$. In the first stage we can take the first 3 axioms and make elimination rule on them, then the next 3 and so on. As a result we will have $3^{k-1}$ conjuncts without $p_{n}$ variable. Then on next stage we will eliminate $p_{n-1}$ in same way. Consequentially eliminating all variables we will have tree like proof with height $n+1$, where each node of tree will be one conjunct which is result of elimination rule of 3 conjuncts from previous level. Let number of levels of tree like proof be from 0 to $n$ (all conjuncts on the level of number 0 have size $n$, the empty conjunct is on the last level with number $n)$. Let $c_{l}(1 \leq l \leq n)$ be one of conjuncts on level $l$ of tree like proof, it is result of elimination rule on 3 conjuncts $c^{\prime}, c^{\prime \prime}$ and $c^{\prime \prime \prime}$ from level $l-1$. By proving $c^{\prime}, c^{\prime \prime}$ and $c^{\prime \prime \prime}$ separately we will have the following $s\left(c_{l}\right)$ space usage for proving $c_{l}$ in above described tree like proof:

$$
s\left(c_{l}\right)=s\left(c^{\prime}\right)+\left|c^{\prime \prime}\right|+\left|c^{\prime \prime \prime}\right|=\left|c^{\prime}\right|+\left|c^{\prime \prime}\right|+s\left(c^{\prime \prime \prime}\right)=\left|c^{\prime}\right|+s\left(c^{\prime \prime}\right)+\left|c^{\prime \prime \prime}\right| .
$$

All conjuncts on the same level $l$ of tree like proof have same size $n-l$. So above equation will look like this:

$$
s\left(c_{l}\right)=s\left(c^{\prime}\right)+2(n-(l-1)) .
$$

As all conjuncts on same level have same space usage, we denote by $S(l)$ the space used for each conjunct on level $l$ :

$$
S(l)=S(l-1)+2(n-l+1)
$$

Total space usage will be space usage on level $n$ :

$$
s \leq S(n)=S(n-1)+2=S(n-2)+2(2+1)=\ldots=2(1+2+\ldots+n)=O\left(n^{2}\right) .
$$

Using the fact that at least 3 determinative conjunct must be in every proof, we have $s_{\varphi}=\Omega(n)$.

The analogous result for more-value logics can be proved also.

Main Theorem

There exists a sequence of 3-tautologies $\varphi_{n}$, for the proof complexity measures of which both in the systems $\mathrm{CN}_{3}$ and $\mathrm{CN}_{3}$-cut-free are valid the following equations: 
1) $\log _{3}\left(\varphi_{n}\right)=\theta(n)$;

2) $\log _{3} \log _{3}\left(t\left(\varphi_{n}\right)\right)=\theta(n)$;

3) $\log _{3} \log _{3}\left(l\left(\varphi_{n}\right)\right)=\theta(n)$;

4) $\log _{3}\left(s\left(\varphi_{n}\right)\right)=\theta(n)$;

5) $\log _{3}\left(w\left(\varphi_{n}\right)\right)=\theta(n)$.

Proof. As $\varphi$ we take the formulas $T T M_{n, m}$ for every $n \geq 1$ and $m=3^{[n / 3]}$. For upper bounds we use the perfect DNF of $\varphi$, and for lower bounds - the properties of $\varphi_{n}$-determinative conjuncts.

It is not difficult to see, that number of variables of $\varphi_{n}$ is $n 3^{n} 3^{[n / 3]}$, the minimal number of variables in every $\varphi_{n}$-determinative conjuncts is $3^{[n / 3]}$, therefore by Remark 1 in the end of point 2.2 the minimal number of $\varphi_{n}$-determinative conjuncts is $3^{3^{\left[n^{n / 3}\right]}}$, hence the number of axioms, using in the system $\mathrm{CN}_{3}$, must be at least $3^{3^{\left[n^{n / 3}\right]}}$.

So, using these statements and Lemma 3, we can obtain all the upper and lower bounds for the system $\mathrm{CN}_{3}$, and using Lemma 2, for the system $\mathrm{CN}_{3}$-cut-free.

Corollary 4. If we take the analogous formulas for k-valued logics for every $n \geq 1$ and $m=k^{[n / k]}$, then the analogous results can be obtained for more valued logics:

There exists a sequence of k-tautologies $(\mathrm{k} \geqslant 4) \varphi_{n}$ in both $\mathrm{CN}_{\mathrm{k}}$ and $\mathrm{CN}_{\mathrm{k}}$-cut-free systems, where the following equations are valid for the proof complexity measures:

1) $\log _{k}\left(\varphi_{n}\right)=\theta(n)$

2) $\log _{k} \log _{k}\left(t\left(\varphi_{n}\right)\right)=\theta(n)$;

3) $\log _{k} \log _{k}\left(l\left(\varphi_{n}\right)\right)=\theta(n)$;

4) $\log _{k}\left(s\left(\varphi_{n}\right)\right)=\theta(n)$;

5) $\log _{k}\left(w\left(\varphi_{n}\right)\right)=\theta(n)$.

Proof is given as above.

\section{Conclusion}

As many-valued logics have many interesting applications, then the proof complexities research in them is very important. In this paper some results of An. Chubaryan [2] are generalized for some version of $\mathrm{k}$ valued logics $(k \geq 3)$. Some class of $\mathrm{k}$-valued $(k \geq 3)$ tautologies are described and the main proof complexity characteristics in two types of proof systems for suggested version of many valued propositional logic are investigated. We hope that analogous results can be proved for other versions of $\mathrm{k}$-valued logics $(k \geq 3)$ also.

Acknowledgments. I am grateful to Professor of YSU and RAU Anahit Chubaryan, supervisor of my $\mathrm{PhD}$ 's thesis for her encouragement and productive discussions, for answering to many of questions about many-valued logics and for helpful suggestions, which allowed me to improve and extend the results.

\section{References}

1. J. Lukasiewicz, O Logice Trojwartosciowej (On three-valued logic), Ruch Filozoficzny (Lwow), Vol.5, 1920, 169171.

2. An.Chubaryan, Relative efficiency of some proof systems for classical propositional logic, Proceedings of NASA RA, Vol.37,N5,2002, and Journal of CMA (AAS), Vol.37, N5, 2002, 71-84. 
3. A.A.Chubaryan, A.S.Tshitoyan, A.A.Khamisyan, On some proof systems for many-valued logics and on proof complexities in it, (in Russian) Reports of NASA RA, Vol.116, N2, 2016, 18-24.

4. Chubaryan Anahit, Khamisyan Artur, Generalization of Kalmar's proof of deducibility in two valued propositional logic into many valued logic, Pure and Applied Mathematics Journal, Vol 6, No. 2, 2017, doi: 10.116448/j.pamj. 20170602.12.,71-75.

5. Y. Filmus, M. Lauria, J. Nordstrom, N. Thapen, N. Ron-Zewi: Space Complexity in Polynomial Calculus, 2012 IEEE Conference on Computational Complexity (CCC), 2012, 334-344. 